\title{
How do people with dementia make sense of their medications? An interpretative phenomenological analysis study
}

Article

Published Version

Creative Commons: Attribution 4.0 (CC-BY)

Open Access

Lim, R. H. M. ORCID: https://orcid.org/0000-0003-1705-1480, Sharmeen, T. and Donyai, P. ORCID: https://orcid.org/00000001-5403-6170 (2022) How do people with dementia make sense of their medications? An interpretative phenomenological analysis study. International Journal of Geriatric Psychiatry, 37 (2). ISSN 0885-6230 doi:

https://doi.org/10.1002/gps.5673 Available at https://centaur.reading.ac.uk/101983/

It is advisable to refer to the publisher's version if you intend to cite from the work. See Guidance on citing.

To link to this article DOI: http://dx.doi.org/10.1002/gps.5673

Publisher: Wiley

All outputs in CentAUR are protected by Intellectual Property Rights law, including copyright law. Copyright and IPR is retained by the creators or other copyright holders. Terms and conditions for use of this material are defined in the End User Agreement. 


\section{CentAUR}

Central Archive at the University of Reading

Reading's research outputs online 


\title{
How do people with dementia make sense of their medications? An Interpretative Phenomenological Analysis study
}

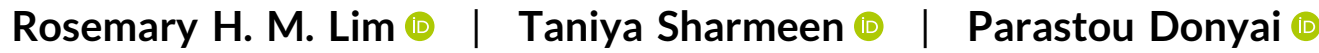

Reading School of Pharmacy, University of Reading, Reading, Berkshire, UK

\section{Correspondence}

Rosemary H. M. Lim, Reading School of Pharmacy, University of Reading, Reading, Berkshire RG6 6DZ, UK.

Email: r.h.m.lim@reading.ac.uk

Funding information

Wellcome Trust, Grant/Award Number: 108320/Z/15/Z

\begin{abstract}
Background: Managing medication is complex and multifaceted for people with dementia and their family carers. Despite efforts to support medication management, medication errors and medication-related hospital admissions still occur. This study investigated how people with dementia viewed and talked about their different medications and their medication taking.

Methods: An interpretative phenomenological analysis (IPA) qualitative research design combining photo elicitation and in-depth interviews was used. People with a diagnosis of mild or moderate dementia took photographs of anything they viewed to be related to medication, with or without the help of family carers, over any twoday period. The photographs were then used as cues for subsequent in-depth interviews, which were analysed using IPA.

Results: Twelve people with dementia were interviewed. Four themes encapsulated the experiences: (1) Medication as a lifeline, (2) Managing medications dominates daily lives and plans, (3) Struggling with uncertainty about the effectiveness of dementia medication and (4) Sense of 'being' and being in control. People with dementia viewed medication as a lifeline, especially donepezil, giving it preference over other daily medication they were using. Managing medications dominated the daily lives and plans of people with dementia and changed the way they viewed themselves and their life. People with dementia continually struggled with the imperceptible benefits of donepezil on their dementia, but despite such uncertainties, continued to take donepezil.

Conclusions: This study provided unique insights into how people with dementia made sense of their medication. Healthcare professionals can use these insights to shape their practice around medication prescribing and advice in dementia. The findings are also useful to researchers looking to develop interventions to support medication management within the home setting.
\end{abstract}




\section{KEYWORDS}

Alzheimer's disease, dementia, Interpretative Phenomenological Analysis, medication, qualitative

\section{Key points}

- There is a dearth of literature focusing on how people make sense of all their medications, within the context of a dementia diagnosis

- We engaged people with dementia, living in their own home, in our study using an Interpretative Phenomenological Analysis qualitative research design combining photo elicitation and in-depth interviews

- We found people with dementia viewed medication, specifically donepezil, as a lifeline, giving it preference over other daily medication they were using

- Managing medications dominated the daily lives and plans of people with dementia and changed the way they viewed themselves and their life

- People with dementia continually struggled with the imperceptible benefits of donepezil on their dementia, but despite such uncertainties, continued to take donepezil

- These insights can help healthcare professionals shape their current practice around medication prescribing and advice, and researchers to develop interventions to support medication management within the home setting

\section{1 | INTRODUCTION}

Dementia is a major global challenge. Worldwide, 46.8 million people live with dementia and this figure is projected to increase to 74.7 million and cost $\$ 2$ trillion per annum by 2030 . $^{1}$ In the United Kingdom, the prevalence of dementia is estimated to double from 850,000 to 1.6 million people and cost $£ 94.1$ bn per annum by $2040 .^{2}$ The impact of dementia extends beyond economics and includes the health, social and emotional lives of individuals, their families and wider society. ${ }^{3-5}$ More than $90 \%$ of people with dementia live with at least one other health condition ${ }^{6}$ and with no cure for dementia on the horizon, medication plays a central role in managing the symptoms of dementia ${ }^{7}$ and the treatment of other existing comorbidities.

Around $61 \%$ of people with dementia live at home ${ }^{2}$ where medication is a part of daily living. As a result, people with dementia and family carers have developed their own strategies for managing medication. ${ }^{8-11}$ There are also interventions to improve medication management, ${ }^{12}$ primarily rationalising the prescribing of psychotropic medication. Managing medication, however, remains complex and multifaceted for people with dementia and family carers ${ }^{10,11}$ and can lead to medication errors and medication-related hospital admissions. ${ }^{13}$ Figures show adherence rates in this group range from $17 \%$ to $100 \% .{ }^{13}$ What is more, people with dementia are three times more likely to be hospitalised due to medication misadventure ${ }^{14}$ and when discharged, they have a two to threefold increased risk of taking either $30 \%$ less or $20 \%$ more of their prescribed medication. ${ }^{15}$ Arguably, empowering this patient group to better manage medication at home should be a priority for health practitioners.

To provide help that is useful to them, it is important to understand how people with dementia themselves, in addition to their family carers, make sense of all their prescribed medication, which in turn influences how they manage their medications within the home setting. Although there have been studies exploring how people with dementia talk and make sense of their diagnosis ${ }^{16,17}$ and dementia medication, ${ }^{18,19}$ there is a dearth of literature focusing on how people make sense of all their medications, within the context of a dementia diagnosis. Personal accounts of experiences provide an important perspective; they not only give people a voice but also provide an important perspective to inform the development of support structures to enable people with dementia to live well with medications. The aim of this study was to describe in-depth, how people with dementia viewed and talked about their different medications and their medication-taking, whether or not the medications were related to their dementia.

\section{2 | METHODS}

\subsection{Study design}

An Interpretative Phenomenological Analysis (IPA) ${ }^{20}$ qualitative research design combining photo elicitation ${ }^{21}$ and in-depth interviews was used. Photo-elicitation is a qualitative interview technique widely used in psychology and some social science disciplines where researchers seek to explore and understand responses, perceptions, reactions, world view, and insights from participants by using photographs or other images as stimuli. Pictures used as stimuli to obtain qualitative data can be researcher-generated or participant-generated and each has particular benefits and challenges. ${ }^{22}$ Participant-generated pictures were used in this photo elicitation interview study. The rationale 
was to directly involve people with dementia in co-producing research about their experiences, without being directed by researchers or others about what to say. Interview transcripts were analysed to explore how people with dementia ascribe meaning to medications and their use of them. The epistemological stance of IPA is that it is possible to gain insight into an individual's cognitive inner world. A researcher using IPA does this through a thorough and systematic interpretation of the dataset, paying close attention to each person's unique detailed accounts. ${ }^{20}$

\section{2 | Participant selection}

Participants on the Berkshire Healthcare NHS Foundation Trust Research Interested List (a list of people with dementia and caregiver volunteers who were interested in taking part in dementia research) that met the inclusion criteria (see Box 1 for details) were invited to participate in the study. Determining a sample size for this study that focused on lived experiences was not straightforward. ${ }^{23}$ When deciding on sample size, we were guided by Geertz (1973), ${ }^{24}$ Smith et al. (2009), ${ }^{20}$ Creswell $(2012)^{25}$ and Alase $(2017)^{26}$ who recommended a range of 2-20 participants. To meet the study aims, 15 people with dementia were anticipated to be enough to generate rich and meaningful analysis. Trained researchers at the Berkshire Memory and Cognition Research Centre (BMCRC) identified recruited and consented participants.

\section{BOX 1: Study inclusion criteria}

- Had a clinical diagnosis of mild or moderate Alzheimer's Disease or mixed dementia with an Alzheimer's Disease component identified in their medical notes

- Montreal-Cognitive Assessment (MoCA) score of nine or above conducted by trained researchers in the BMCRC to indicate the severity of the dementia condition

- Prescribed at least one regular medication of any type, which may or may not include an acetyl cholinesterase inhibitor such as donepezil

- Live in their own home

- Receive some form of help with their medication from one or more family carer(s)

- Capacity to consent (based on assessment by trained researchers)

\section{3 | Setting}

An experienced female qualitative researcher with a background in anthropology and health research (TS) conducted face-to-face interviews at each participant's home at a mutually suitable time.

\subsection{Data collection}

The researchers had no prior relationship with any of the participants. Participants were co-creators of research knowledge; they were loaned a digital camera and asked to take photographs of anything (e.g., objects and places not limited to their own home) that they viewed to be related to medication and medicationtaking, over any two-day period. Verbal and written guidance were provided. The photographs were then used as cues for subsequent in-depth interviews ${ }^{27}$-see Box 2 for the interview topic guide and Supplementary Information S1 for an excerpt of the interview transcript with photographs. Interviews were digitally audio-recorded and transcribed verbatim. To ensure participants were comfortable, some interviews took place with the family carer present forming participant-carer dyads. In these interviews, we maintained our focus on encouraging participants to share their personal experiences but did not stop carers from participating in the interview had they wished to; carer contributions were generally limited to prompting or clarifying statements made by participants.

\section{BOX 2: Interview topic guide}

Warm up questions

- How did you find using the camera?

- How did you find the process of taking pictures? Were they planned or spontaneous?

Main questions

- Present participant with one picture at a time, and use every picture taken: Can you tell me what this picture means to you? Were you thinking of anything specific when you took the picture?

- General: What place does medication have in your life at this moment? Have you changed the ways you use medication over time? How has medication affected your relationship with others?

Closing

- Is there anything else you want to tell me or add anything to what you have already said?

\section{5 | Data analysis}

Analysis involved familiarization with each interview transcript in conjunction with the original audio recordings and corresponding photographs, making notes on specific aspects of the transcript (descriptive, linguistic and conceptual), interpreting and then 
classifying these notes to develop initial themes (similar clusters of ideas) and subsequently a master list of themes and subthemes to capture the essence of each participant's account. This process was repeated for every transcript on Microsoft Word. A comparison of all the themes and sub-themes for each transcript was made and recurrent patterns across all transcripts identified and consolidated into a list of super-ordinate themes that accounted for the experiences of the sample. Throughout the analytical process, the researchers 'bracketed' their own assumptions, experiences and judgements and kept reflexive notes. Two researchers (TS and RL) independently analysed the entire dataset. RL is a trained qualitative researcher and an academic pharmacist. TS and $\mathrm{RL}$ discussed their analyses to arrive at the themes presented in this paper.

\section{3 | RESULTS}

\section{1 | Participants}

Interviews took place between January-May 2017. Twelve in-depth face-to-face interviews were conducted with people with dementia. Table 1 shows the participant characteristics.

The mean age of participants was 75.2 years (standard deviation [SD] 9.1, range 60-89) and 58.3\% ( $n=7)$ were male. Most participants had received a diagnosis within the previous 5 years $(n=10,83.3 \%$ ). The mean MoCA score was 17.3 (SD 4.4, range 12-25). A score of $18-25$ was graded as mild cognitive impairment and $10-17$ as moderate cognitive impairment. Two participants lived on their own but had family caregivers living nearby. A total of 129 photographs were taken, ranging between 3 and 20 per participant. Most photographs were taken independently by the person with dementia without caregiver prompting. Most people with dementia participated meaningfully and independently in the interview, with photographs they had taken used as cues to discuss their experiences. Interviews with participants lasted between 18 and $66 \mathrm{~min}$ (mean $35 \mathrm{~min}$ ).

\section{2 | Interview findings}

In-depth analysis of interviews using IPA generated four main themes: (1) Medication as a lifeline, (2) Managing medications dominates daily lives and plans, (3) Struggling with the uncertainty about the effectiveness of dementia medication and (4) Sense of 'being' and being in control. Figure 1 shows the relationship between the four inter-related themes.

Theme 1: Medication as a lifeline: 'Without it [medication], life would be very difficult or impossible'
TABLE 1 Demographic details of participants $(n=12)$

Age (years)

$60-69$

$4(33.3 \%)$

70-79

$4(33.3 \%)$

80-89

Gender

Female

Male

$7(58.3 \%)$

Diagnosis

Alzheimer's disease

$10(83.3 \%)$

Mixed dementia

$2(16.7 \%)$

Years since diagnosis

$0-2$

$7(58.3 \%)$

3-5

$3(25.0 \%)$

6-10

$1(8.3 \%)$

$>10$

$1(8.3 \%)$

MoCA score

10-17 (moderate cognitive impairment)

7 (58.3\%)

18-25 (mild cognitive impairment)

$5(41.7 \%)$

Living arrangements

Alone

With a partner/spouse

$10(83.3 \%)$

Participants talked about their experiences from a position of acceptance of their dementia diagnosis and their need for medication.

I know that I wouldn't be probably as I am without the medicine I take on a daily basis. It's not a choice thing. It's a necessity of life I guess, for me. So, I've accepted that and that's it.

Participant 9

As such, medication, specifically their dementia medication donepezil, was viewed as the primary help to manage dementia. Participants acknowledged that donepezil was not a cure, but key in halting the deterioration of dementia. All knew that donepezil was to be taken for life because there is currently no cure. One participant made explicit her own diagnosis of dementia as a terminal condition. She talked about donepezil as part of palliative care.

Well until they can give you new brain cells, that's it really. All they can do is palliative, isn't it really?

Participant 10 


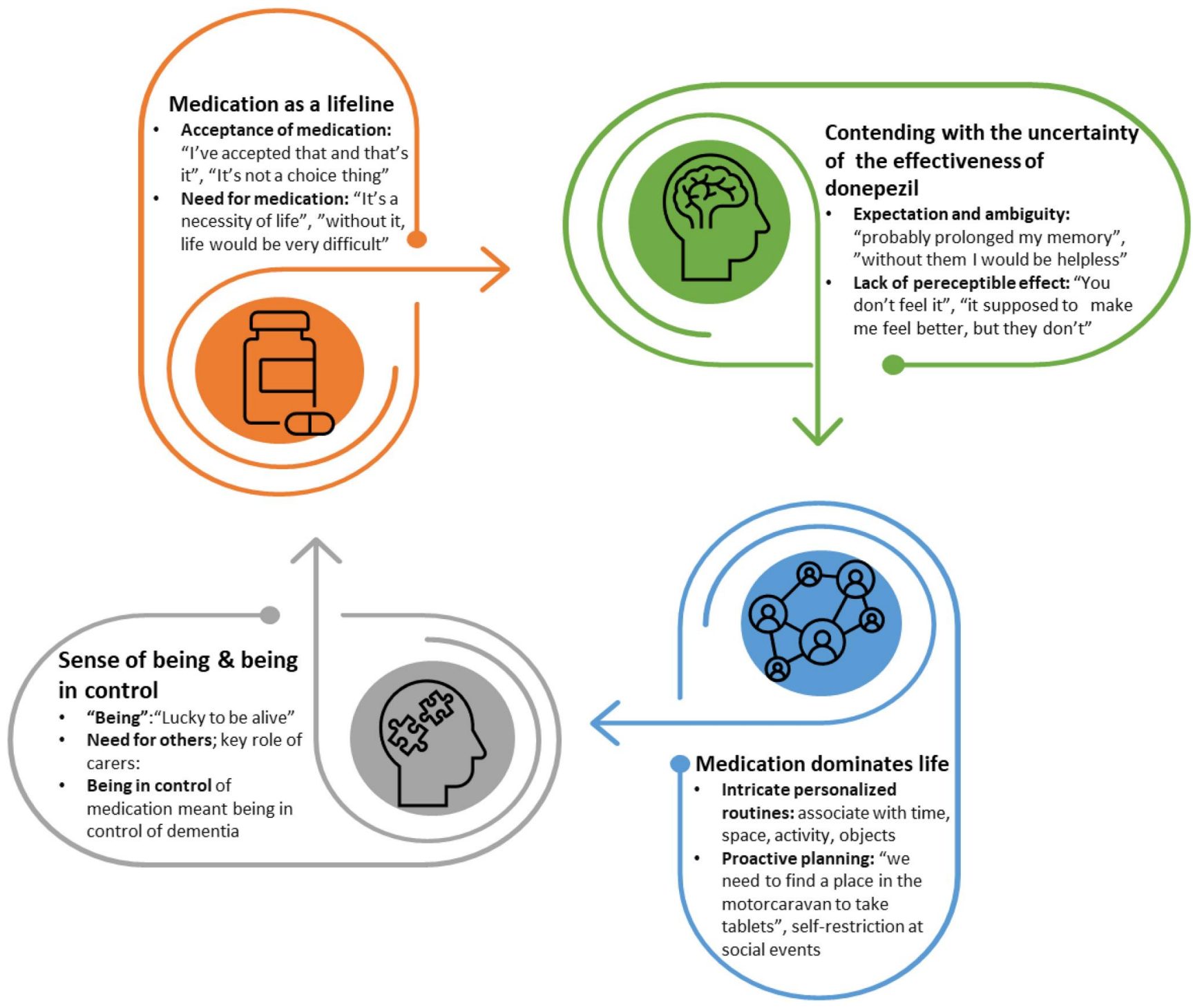

FIGURE 1 Relationship matrix of super-ordinate themes

Donepezil was a lifeline and provided hope for 'more years' with their loved ones. Participants wanted to enjoy life with their family and donepezil played a key part in enabling life to continue.

In one sense. It is one of the key things in my life. Without it life would be very difficult or impossible wouldn't it? Yes. Oh yes, I regard it as a very necessary and important thing that I must be careful with, that I must keep on taking it and taking it at the right time, yes.

\section{Participant 11}

To not take donepezil was to give up on life. Participants wanted to live life to the fullest, for as long as possible and donepezil was a means for that to happen.

I take it [donepezil] because I know it helps me. So, I'm, there's no point me saying I don't want it, I, then I may as well give up... But that helps me to keep going and enjoying life and that's what, that's what I want to do.

Participant 8

Theme 2: Managing medications dominates daily lives and plans

It followed that medication dominated the daily lives and plans of participants. Medication-taking revolved around the establishment and maintenance of an intricate personalised routine within the home. The extent and complexity of the routine differed. Routines were associated with a particular time, space, activity and specific objects. For example, medication in a visible pillbox on the kitchen table was taken at breakfast time. A wide range of helpful objects were used such as compliance aids, alarms, calendars, and record books-these were made clearly visible and easily accessible. These routines were established over time following trial and error (see Box 3 for examples). Participant-carer dyads were strict in following these routines. 


\section{BOX 3: Examples of participant medication management routines}

Participant 1 receives a pre-packed medication blister pack from the community pharmacy. Sometimes she forgets the time and day, so she places a day clock in a prominent place in her living room. She takes her medication once a day at breakfast time.
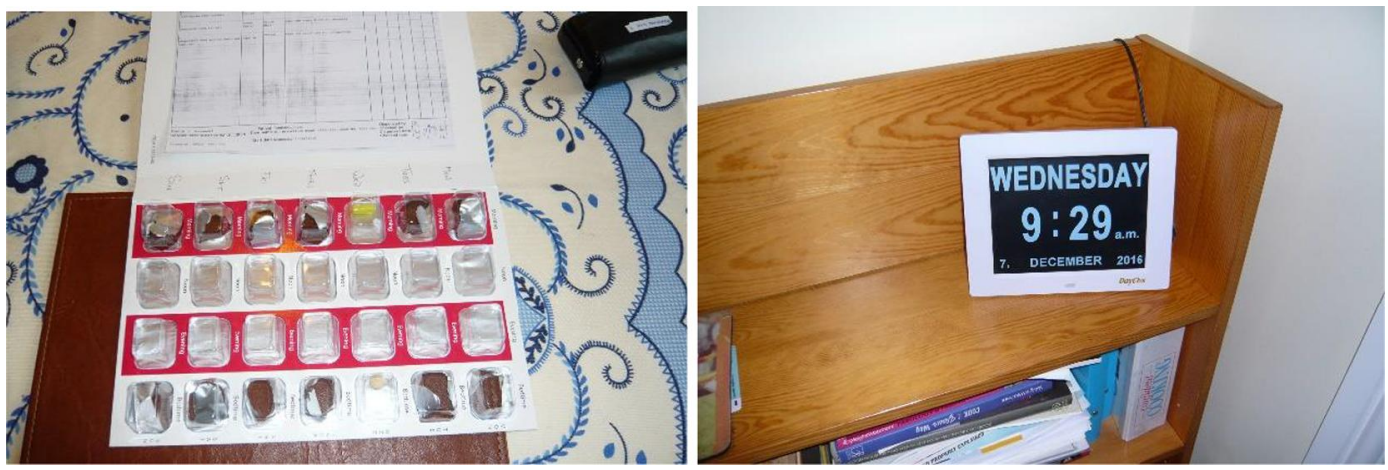

Participant 4 does not use a compliance aid. She places the same red cup on the same red table mat, along with her medication bottle, at a specific corner of the kitchen before she goes to bed every night. This visual arrangement reminds her to take her medication when she enters the kitchen the next morning.
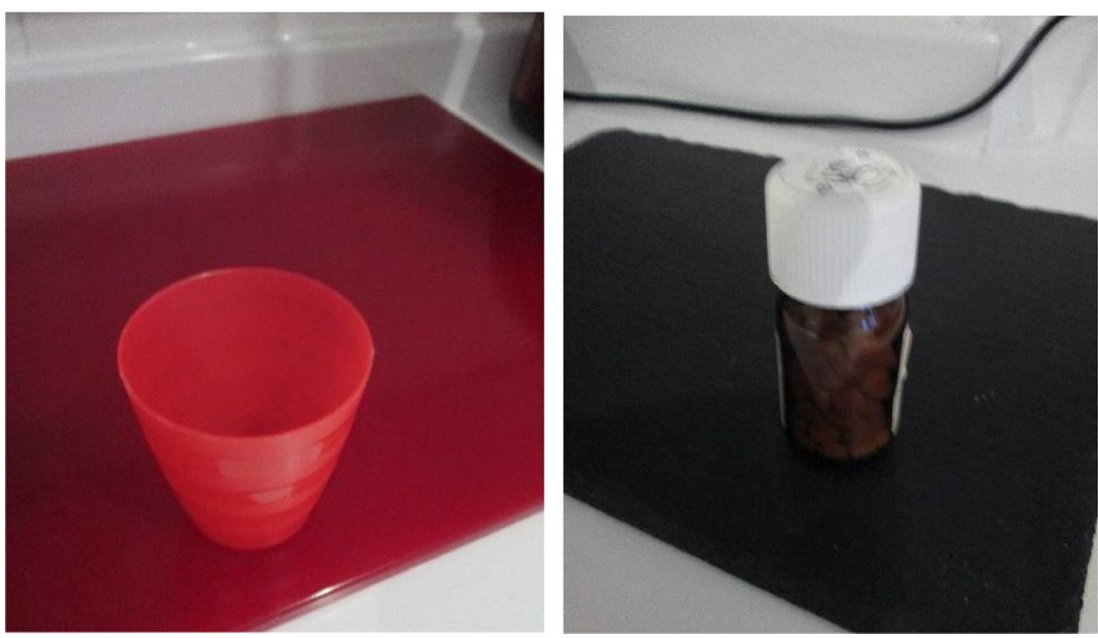

Participant 6 does not use a compliance aid. She and her husband both take medication. All their medications are stored in the same green box. She takes medication once a day, at breakfast, the same time that her husband takes medication.

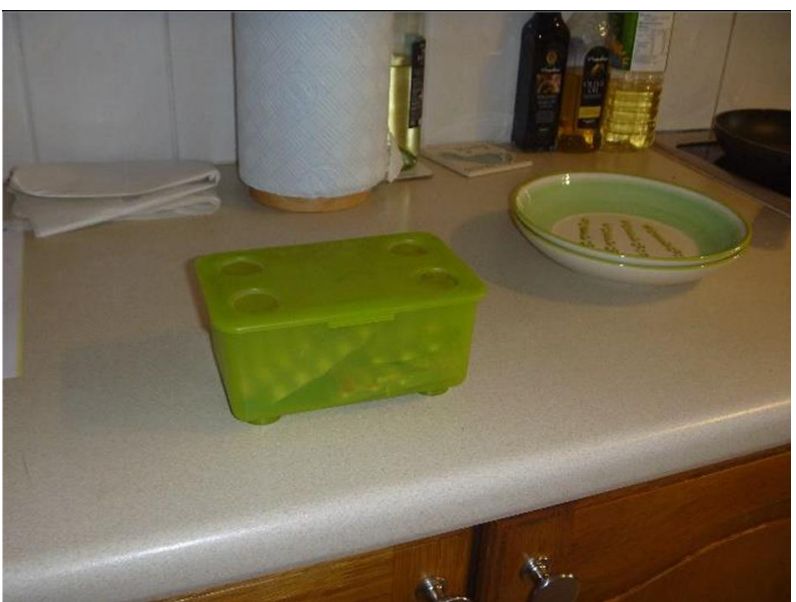

Participant 8 does not use a compliance aid. Due to an incident involving the participant taking an unintentional overdose (he forgot he had already taken his medication so took it again), his wife keeps all his medication in a cashbox. When it is time to take medication i.e. breakfast time, he tells his wife he needs his medication. She then opens the cashbox and takes out the medication. The participant then takes the medication. He marks on a paper calendar placed in the kitchen that he has taken his medication. 


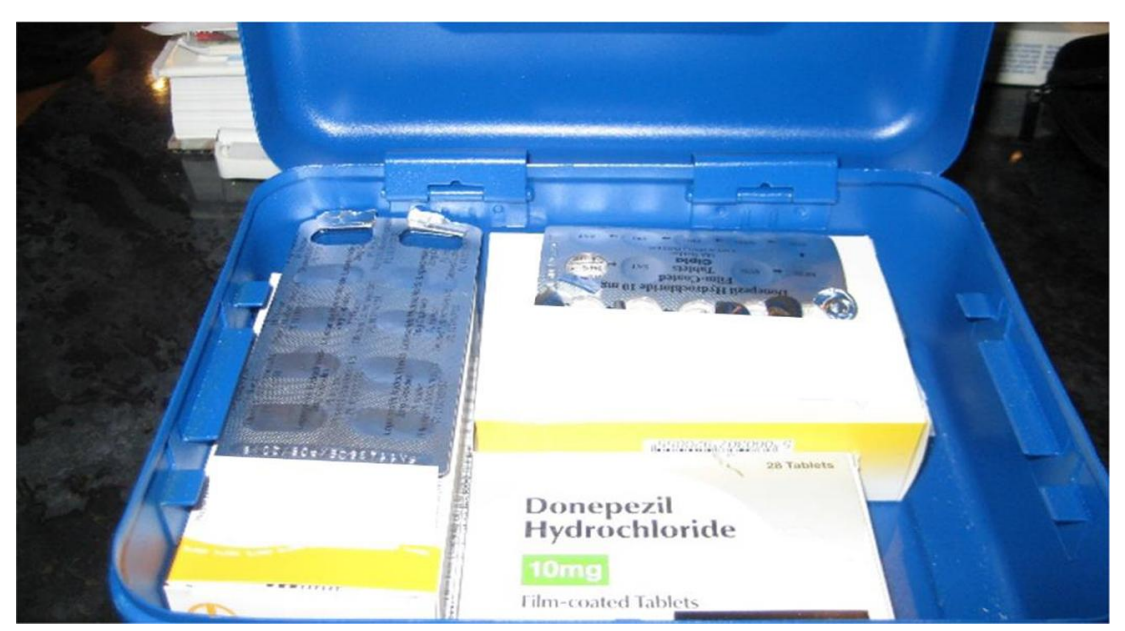

Participant 12 refills his dosette box every Monday at 8 AM. He is prescribed a range of medication. Immediately after his morning wash, he takes one medication, a tablet, removed from an original pack, in the bathroom. He says there is no space in his dosette box, so he keeps it in the bathroom and takes it in the morning. Then, at breakfast time, he takes three more tablets; he removes them from his dosette box and places them in a special small ceramic bowl that he associates with medication. As for donepezil, this is kept in the original box. He takes donepezil with a main meal, either in the afternoon or in the evening. As he often visits his partner in the flat next door and sometimes have a main meal there, he keeps an additional store of donepezil in her flat.
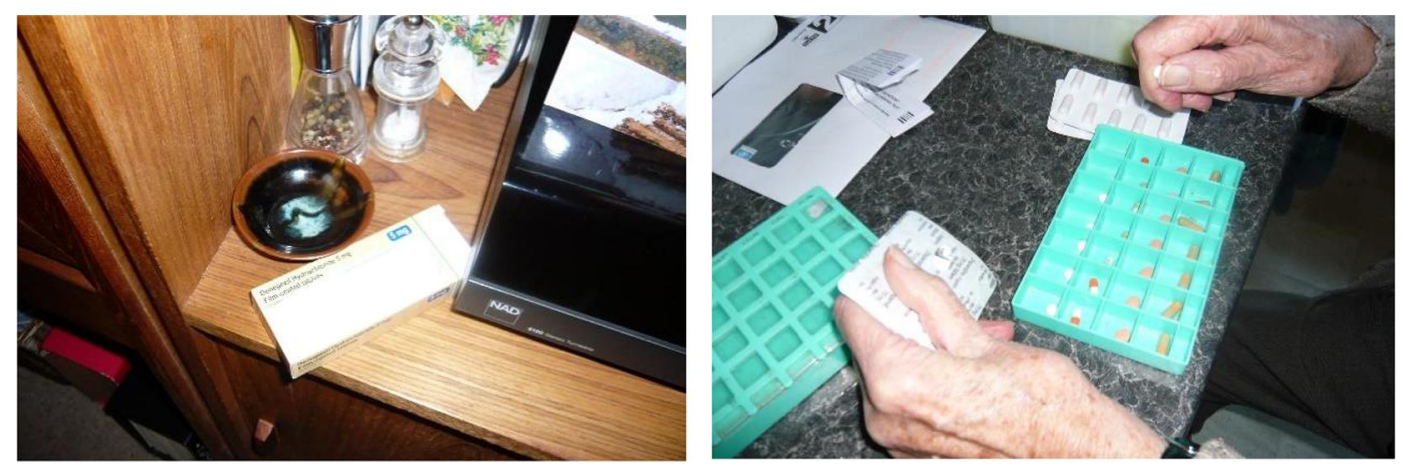

An established routine was key in helping participants take their medication as independently as possible. Social events and holidays however, required further proactive planning with medication-taking. For one participant who took a motorcaravan on holiday, the inside of this vehicle was set up to be almost identical to their kitchen at home to recreate a familiar environment associated with medication-taking. One participant poignantly talked about his experience of restricting himself from drinking wine during "card night" with his family because he was on medication (see Figure 2).

Right this picture, it's obviously, someone has had a glass of wine, there's some crisps there...It probably meant that if we were drinking wine I shouldn't drink too much of it because I need to take the tablets.

Participant 5 talking about Figure 2

Theme 3: Struggling with uncertainty about the effectiveness of dementia medication
Although participants did not view donepezil as a cure for dementia, they expected it to have some effect on their dementia; positive or negative. When talking about these effects, however, responses were tentative.

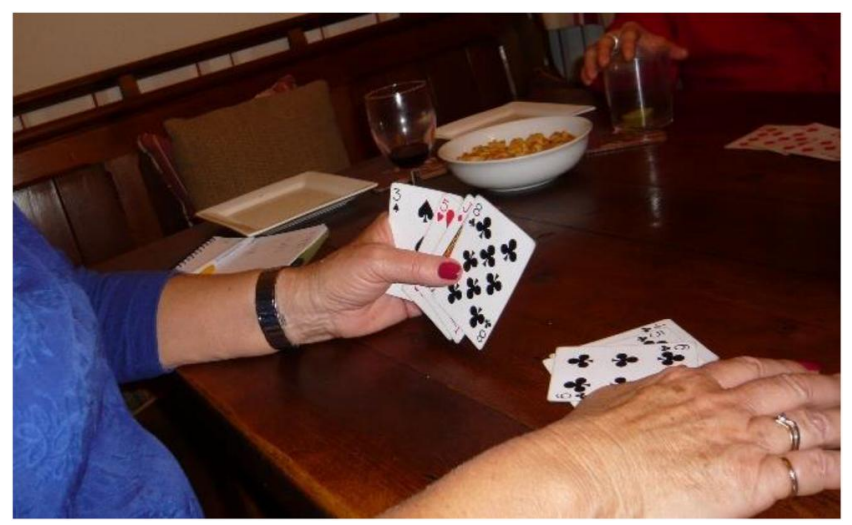

FIGURE 2 A picture taken by Participant 5 when asked to capture views about medication and medication-taking 
I'm grateful that these things have been developed because I know that they have probably prolonged my memory, if I were without them, I would be pretty helpless I think.

Participant 12

I guess I think I've lived a bit longer maybe, taking all this medicine. I'm not sure.

Participant 9

Participants' views about the effectiveness of donepezil were tentative because they saw no changes in cognitive test results, could not perceive relief of their physical symptoms or did not associate a positive emotional response from taking donepezil. This was contrasted with taking other medications such as aspirin for a headache or lansoprazole for stomach pains; with those, the removal of pain would indicate that the medication had taken effect but not so for donepezil.

It's [picture of Venice], reminds me of holidays and relaxation which the tablets [donepezil] were supposed to give me same pleasure, make me feel better but they don't.

Participant 5

Despite the uncertain and imperceptible effects of donepezil, participants were determined to continue taking it; the risk of a deteriorating condition was too great.

\section{Theme 4: Sense of 'being' and being in control}

Participants showed insight when they talked about themselves as someone with a diagnosis of dementia. There was a strong sense of 'being'; they talked about being grateful for being alive and for their life.

Yeah, another day outside with the sun shining out there, thinking I'm lucky to be alive.

\section{Participant 5}

There was clear acknowledgement across all participants that they needed others to journey with them, living with dementia. Several showed empathy towards their carer.

I mean it must be irritating at times when you've told me something and then you, half an hour later or perhaps an hour or two later you will say, well I told you that, and I'll say oh yes, sorry, yes, of course you did.

\section{Participant 12}

Participant: When you hear that large thumping noise in the bath which does not happen very often, but that's me falling over.
Carer: Oh well. But anyway, we can mention it to the doctor when we go next, we have already.

Participant: Well you've put up with a lot haven't you?

Participant 7

The relationship between the participant and carer was crucial in determining the participants' sense of self. Most participants had carers who were empathic, encouraging, patient and kind. These carers played a key role in 'scaffolding' the participant to enable them to be as independent as possible in managing medications. They 'engineered' the environment with and for the participant to take their medications. This resulted in participants being able to manage their own medications as independently as possible within a (carer-) controlled environment. Their ability to manage their own medications, that is, being in control, was key to their sense and view of self, and their wellbeing. Being in control of their medications meant being in control of their dementia.

There it is. Well that's it. I think it's absolutely brilliant and you can see what it's called. PPPP, and I just think it's great that I can tick everything off so easily, yeah, and yeah, it's made all the difference, because I'd never have remembered otherwise, would I?

Participant 2

Oh I'm quite pleased with that really. I'm not, I don't know, maybe I'm doing myself down a bit but I've always thought I'm not the most organised person in the world. But with this, I'm organised so that's comforting.

Participant 10

Managing medications can also cause stress and tension within the participant-carer dyads and this was often due to differing concerns and priorities. Difficult relationships between participants and their family caregivers were due to past negative experiences such as an accidental medication overdose. In these cases, carers took over all or most of the medication management process, for fear of potential harm to the participant and deterioration of their dementia. The need to manage medications led to a change in dynamics within the participant-carer dyad and as a result some participants viewed and talked about themselves differently, both positively and negatively.

Participant: Do you think I'm a simpleton? [angry voice]

Carer: Course I don't think you're a simpleton. I know you act like one but

Participant: I know. I take five in the morning and two at night. Is that correct?

Carer: No. But that's all right.

Participant: Right so what do I take then?

Participant 9 
Most participants were careful to only take medications prescribed by the doctor. But for one participant, the carer researched a combination of medication and vitamins that could help dementia and strongly suggested the participant take them, which the participant agreed to, against the consultant doctor's advice.

I researched, in America, to do with dementia a dose, small dose of folic acid, B6 and B12 daily helps, there's been a small research, they've done quite a few small studies, they haven't done big studies... and I put you on that, and $\operatorname{Dr}$ (name), your doctor, she didn't want us to go on that, she said, no don't do that...But I wasn't interested in that, I wanted as many good things going in..[for the participant to take it].

Carer to Participant 4

\section{DISCUSSION}

This study provided unique insights into the views of people with dementia about their medication. Our principal findings were that people with dementia viewed medication, specifically donepezil, as a lifeline, giving it preference over other daily medication they were using. Managing medications dominated the daily lives and plans of people with dementia and changed the way they viewed themselves and their life. People with dementia continually struggled with the imperceptible benefits of donepezil on their dementia, but despite such uncertainties, continued to take donepezil.

Being diagnosed with dementia is a life-changing event. Although non-pharmacological interventions are available to promote cognition, independence and wellbeing, ${ }^{28}$ people with dementia viewed donepezil as the cornerstone for managing their condition, reflecting a biomedical view of health. People with dementia were aware that their condition was incurable. Some described their condition as terminal and their current treatment, palliative. Despite this, donepezil was viewed as a lifeline to help with dementia symptoms. The participants would take their medication, specifically donepezil, religiously despite the uncertainty of its effectiveness in managing dementia symptoms. This apparent lack of efficacy is unsettling and problematic. But, due to there being no cure for dementia, participants viewed the risk of deterioration to be greater than the burden and issues related to managing medications. Thus, they continued to take donepezil. These findings were consistent with Hutchings et al.'s ${ }^{18}$ study, reporting varied experiences of people with dementia taking cholinesterase inhibitors. Most of the people we interviewed had co-morbidities but some viewed and described donepezil as being more important than medications prescribed for other conditions. Our findings aligned with Lindstrom et al.'s ${ }^{19}$ study where people with dementia and carers expressed relatively high hopes for medications intended to help with memory loss. Our findings however, contrasted with Rathbone's $(2020)^{29}$ who found that experiences of medication-taking were similar amongst patients who had so-called asymptomatic conditions, for example, hypertension and those with symptomatic conditions, for example, respiratory disease. Views from people with dementia in this study also contrasted with those of people with other terminal illnesses who are reported to undergo a 'transition' period during which they place less importance on certain types of medication. ${ }^{30}$

The place that medications have in the lives of people with dementia and their family carers was reflected in the way medication dominated their lives. There is increasing understanding of how people with dementia manage their medications ${ }^{10,11}$ highlighting the complexity and all-consuming nature of the task. This current study, however, has broadened our understanding of the drivers that lead people with dementia and family carers to persevere with the medications through the development of a range of intricate, personalised routines. The 'success' of each routine was usually heavily dependent on the family carer, consistent with previous studies. ${ }^{9,31,32}$ A change in the dynamics of the dyadic relationship occurs because of the need to manage medications and comes to redefine how the person with dementia views themselves, whether positive or negative. The reciprocal dyadic relationship between the carer-participant was vital in establishing 'sense of self' among the participants, consistent with Tuomola et al.'s work. ${ }^{33}$

\section{1 | Implications for practice and future research}

Understanding how people with dementia view medications is key in shaping conversations around treatment options. Healthcare professionals should continue to talk with people with dementia and their carers during consultations about the medications prescribed, the potential benefits, side effects and how they will be followed up. These conversations are important and can contribute to people with dementia and carers' assumptions and expectations about medications. How people with dementia and family carers view medications can influence their understanding about the impact of their medications and therefore potentially their wellbeing. Clear communication around monitoring and frequency of monitoring of dementia symptoms is key to alleviate doubts and worry and to ensure medications are taken safely.

There appear to be few theory-driven interventions specifically designed to support people with dementia and family carers to manage medications within the home setting. Interventions such as reducing psychotropic drug use ${ }^{12}$ and bespoke strategies developed by family carers and people with dementia ${ }^{11}$ exist. But these have been piecemeal focusing on specific and often limited aspects of medication management. There is then arguably a place for theorydriven interventions to support people with dementia and family carers manage medications daily within their homes that explicitly uses knowledge and experiences of people with dementia and carers' specific contextual environment. The findings of this study could be used to inform the development of formal interventions to be tested in future studies. 


\subsection{Strengths and limitations}

There is limited in-depth exploration of the lived experiences of people with dementia and specifically their views on medications. Our original study adds to this body of knowledge through careful and sensitive elicitation of knowledge, co-produced with people with dementia. The use of pictures, taken by people with dementia, had a two-fold advantage. First, giving control to people with dementia to 'set the agenda' of the interview and secondly as cues to trigger their memory when sharing their lived experiences. Photo elicitation is not new in dementia research but not often used in the context of medication management. The methodological approach could be of relevance to researchers in countries across the world in eliciting culturally specific lived experiences. The design of IPA studies focuses on obtaining 'rich', 'deep' and 'thick descriptions' of the 'lived experiences' of homogenous participants to have 'better understanding' of the overall perceptions among the participants' lived experiences. In line with this, our participants were homogeneous in terms of ethnicity; they were all white. Participants from different ethnic and cultural backgrounds may have different views of medication.

\section{5 | CONCLUSION}

This study provided unique insights into the views of people with dementia in terms of their medication. Dementia medication, donepezil, was viewed as a lifeline despite its imperceptible effects and reflects the uncertainty of living with dementia. Healthcare professionals can use these insights to shape their current practice around medication prescribing and advice. Future work can focus on using these insights to inform intervention development work to support medication management within the home setting.

\section{ACKNOWLEDGEMENTS}

The study would not have been possible without all the people with dementia and family carers who took part in the study-thank you. We thank the Berkshire Memory and Cognition Research Centre (BMCRC) for recruiting and consenting participants. The study was funded by The Wellcome Trust Seed Award in $\mathrm{Hu}-$ manities and Social Science awarded to RL (PI) and PD (grant number $108320 / Z / 15 / Z)$. The funders had no role in the design of the study, the collection and analysis of the data or in the preparation of the manuscript.

\section{CONFLICT OF INTEREST}

The authors declare that they have no competing interests.

\section{ETHICAL APPROVAL}

The study received ethical approvals from the United Kingdom National Health Service Health Research Authority (IRAS ID 200310), the English South East Coast-Surrey Research Ethics
Committee (reference: 16/LO/1574) and the University of Reading Research Ethics Committee (reference: 16/57). The study was conducted in accordance with the relevant ethical guidelines as set out by the ethics committees. All participants provided written informed consent prior to participation in the study. Participants were informed prior to and during the consent-taking process that their participation was voluntary and that they were free to withdraw from the study should they wish to without prejudice.

\section{AUTHORS CONTRIBUTIONS}

Rosemary H. M. Lim and Parastou Donyai conceived the study. Rosemary H. M. Lim and Parastou Donyai secured funding for the study. Rosemary H. M. Lim, Taniya Sharmeen and Parastou Donyai contributed to the study design. Taniya Sharmeen conducted the interviews. Taniya Sharmeen and Rosemary H. M. Lim analysed the study data. Rosemary H. M. Lim drafted the article. Taniya Sharmeen and Parastou Donyai revised the manuscript critically for intellectual content. All authors agreed and approved the final manuscript.

\section{CONSENT FOR PUBLICATION}

All participants provided written consent for photographs taken as part of the study, and anonymised portions of transcripts, to be used in publications.

\section{DATA AVAILABILITY STATEMENT}

The datasets used and/or analysed during the current study are available from the corresponding author on reasonable request.

\section{ORCID}

Rosemary H. M. Lim (D) https://orcid.org/0000-0003-1705-1480

Taniya Sharmeen (iD https://orcid.org/0000-0002-4600-0001

Parastou Donyai (D) https://orcid.org/0000-0001-5403-6170

\section{REFERENCES}

1. Alzheimer's Disease International (ADI). World Alzheimer Report 2018 The state of the art of dementia research: New frontiers; 2018.

2. Prince $M$, Knapp M, Guerchet M, et al. Dementia UK: Update Second Edition; 2014.

3. Lewis F, Schaffer SK, Sussex J, et al. Trajectory of Dementia in the UK Making a Difference. London Consulting Report, Office of Health Economics; 2014

4. Farina N, Page TE, Daley S, et al. Factors associated with the quality of life of family carers of people with dementia: a systematic review. Alzheimer's Dementia. 2017;13(5):572-581.

5. Connell J, Page SJ, Sheriff I, Hibbert J. Business engagement in a civil society: transitioning towards a dementia-friendly visitor economy. Tour Manag. 2017;61:110-128.

6. Browne J, Edwards DA, Rhodes KM, Brimicombe DJ, Payne RA. Association of comorbidity and health service usage among patients with dementia in the UK: a population-based study. BMJ Open. 2017; 7:e012546.

7. Donegan K, Fox N, Black N, Livingston G, Banerjee S, Burns A. Trends in diagnosis and treatment for people with dementia in the 
UK from 2005-2015: a longitudinal retrospective cohort study. Lancet. 2017;2(3):e149-e156.

8. Alsaeed D, Jamieson E, Gul MO, Smith FJ. Challenges to optimal medicines use in people living with dementia and their caregivers: a literature review. Int J Pharm. 2016;512(2):396-404.

9. Aston L, Hilton A, Moutela T, Shaw R, Maidment I. Exploring the evidence base for how people with dementia and their informal carers manage their medication in the community: a mixed studies review. BMC Geriatr. 2017;17:242. https://doi.org/10.1186/s12877017-0638-6

10. Barry HE, McGrattan M, Ryan C, et al. Perspectives of communitydwelling people with dementia and their carers about medicines management: a qualitative study. Int J Pharm Pract. 2019;27(S2): 15-16.

11. Lim RH, Sharmeen T. Medicines management issues in dementia and coping strategies used by people living with dementia and family carers: a systematic review. Int J Geriatr Psychiatr. 2018;33(12): 1562-1581.

12. McGrattan $M$, Ryan $C$, Barry $\mathrm{HE}$, Hughes $\mathrm{CM}$. Interventions to improve medicines management for people with dementia: a systematic review. Drugs Aging. 2017;34(12):907-916.

13. Smith D, Lovell J, Weller C, et al. A systematic review of medication non-adherence in persons with dementia or cognitive impairment PLoS One. 2017;12(2):e0170651.

14. Mullan J, Burns P, Mohanan L, Lago L, Jordan M, Potter J. Hospitalisation for medication misadventures among older adults with and without dementia: a 5-year retrospective study. Australas J Ageing. 2019;38:e135-e141. https://doi.org/10.1111/ajag.12712

15. Gray S, Mahoney J, Bough D. Medication adherence in elderly patients receiving home health services following hospital discharge. Ann Pharmacother. 2001;35:539-545.

16. Beard R. In their voices: identity preservation and experiences of Alzheimer's disease. J Ageing Stud. 2004;18:415-428.

17. Johnson HF. Exploring the lived experiences of people with dementia through Interpretative Phenomenological Analysis. Qual Rep. 2016;21:695-711.

18. Hutchings D, Vanoli A, McKeith I, Brotherton S, Mcnamee P, Bond J. Good days and bad days: the lived experience and perceived impact of treatment with cholinesterase inhibitors for Alzheimer's disease in the United Kingdom. Dementia. 2010;9(3): 409-425.

19. Lindstrom H, Smyth K, Sami S, et al. Medication use to treat memory loss in dementia Perspectives of persons with dementia and their caregivers. Dementia. 2006;5(1):27-50.

20. Smith J, Flowers P, Larkin M. Interpretative Phenomenological Analysis: Theory, Method and Research. Sage; 2009.

21. Harper D. Talking about pictures: a case for photo elicitation. Vis Stud. 2002;17(1):13-26. https://doi.org/10.1080/14725860220137345

22. Copes H, Tchoula W, Brookman F, Ragland J. Photo-elicitation interviews with vulnerable populations: practical and ethical considerations. Deviant Behav. 2018;39(4):475-494. https://doi.org/10. 1080/01639625.2017.1407109
23. Sim J, Saunders B, Waterfield J, Kingstone T. Can sample size in qualitative research be determined a priori? Int I Soc Res Methodol. 2018;21:619-634.

24. Geertz C. The Interpretation of Cultures. Basic Books, Inc; 1973.

25. Creswell JW. Educational Research: Planning, Conducting and Evaluating Quantitative and Qualitative Research. 4th ed. Pearson; 2012.

26. Alase A. The Interpretative Phenomenological Analysis (IPA): a guide to a good qualitative research approach. Int J Educ Lit Stud. 2017; 5(2):9-19. https://doi.org/10.7575/aiac.ijels.v.5n.2p.9

27. Alzheimer's Society. Photo Interviews Used to Understand Experience of Managing Medications. Accessed September 4, 2021. https://www. alzheimers.org.uk/dementia-professionals/dementia-experiencetoolkit/real-life-examples/dementia-friendly/photo-interviewsmedications

28. National Institute for Health and Care Excellence (NICE). Dementia: Assessment, Management and Support for People Living with Dementia and Their Carers; 2018. NICE guideline [NG97] https://www.nice.org. uk/guidance/ng97

29. Rathbone A, Jamie K, Todd A, Husband A. A qualitative study exploring the lived experience of medication use in different disease states: linking experiences of disease symptoms to medication adherence. J Clin Pharm Therapeut. 2020;46:352-362. https://doi. org/10.1111/jcpt.13288

30. Todd A, Holmes H, Pearson S, et al. I don't think I'd be frightened if the statins went' : a phenomenological qualitative study exploring medicines use in palliative care patients, carers and healthcare professionals. BMC Palliat care. 2016;15(1):13.

31. Erlen J, Lingler J, Sereika S, Tamres LK, Happ MB, Tang F. Characterizing caregiver mediated medication management in patients with memory loss. J Gerontol Nurs. 2013;39:30-39.

32. Smith F, Grijseels MS, Ryan P, Tobiansky R. Assisting people with dementia with their medicines: experiences of family carers. Int $J$ Pharm Pract. 2015;23(1):44-51. https://doi.org/10.1111/ijpp.12158

33. Tuomola J, Soon J, Fisher P, Yap P. Lived experience of caregivers of persons with dementia and the impact on their sense of self: a qualitative study in Singapore. J Cross Cult Gerontol. 2016;31: 157-172. https://doi.org/10.1007/s10823-016-9287-z

\section{SUPPORTING INFORMATION}

Additional supporting information may be found in the online version of the article at the publisher's website.

How to cite this article: Lim RHM, Sharmeen T, Donyai P. How do people with dementia make sense of their medications? An Interpretative Phenomenological Analysis study. Int J Geriatr Psychiatry. 2022;1-10. https://doi.org/10. 1002/gps.5673 